Voix et Images

\title{
Des Romans bien tranquilles : les prix du Cercle du Livre de France (1960-1965)
}

\section{Jean-Claude Choul et Michel de Smet}

Volume 6, numéro 1, automne 1980

Gilles Marcotte

URI : https://id.erudit.org/iderudit/200254ar

DOI : https://doi.org/10.7202/200254ar

Aller au sommaire du numéro

Éditeur(s)

Les Presses de l'Université du Québec

ISSN

0318-9201 (imprimé)

1705-933X (numérique)

Découvrir la revue

Citer cet article

Choul, J.-C. \& de Smet, M. (1980). Des Romans bien tranquilles : les prix du Cercle du Livre de France (1960-1965). Voix et Images, 6(1), 127-145.

https://doi.org/10.7202/200254ar d'utilisation que vous pouvez consulter en ligne.

https://apropos.erudit.org/fr/usagers/politique-dutilisation/ 


\title{
Des Romans bien tranquilles: les prix du Cercle du Livre de France (1960-1965)
}

\author{
Par Jean-Claude Choul \\ et \\ Michel de Smet
}

\section{L'idéologie et sa lecture}

Si nous avons pu songer un instant que le Prix du Cercle s'articulait à un «système d'idées et de jugements, explicite et généralement organisé, qui sert à décrire, expliquer, interpréter ou justifier la situation d'un groupe ou d'une collectivité et qui, s'inspirant largement de valeurs, propose une orientation précise à l'action historique de ce groupe ou de cette collectivité " 1 , la lecture des romans retenus (de 1960 à 1965, à l'exception de 1962 , où le prix n'a pas été décerné) ${ }^{2}$ nous a fait déchanter. Si tout prix constitue un phénomène idéologique, par son inscription dans un système social de valeurs, s'il organise une lecture de la production littéraire d'une époque, s'il tend à justifier ou à privilégier certains discours, il n'en reste pas moins que la cohérence faisait défaut. En effet, on verra plus loin dans l'examen de chacune des œuvres que si le fonctionnement global est apparenté, c'est qu'il se dévoile comme fonctionnement d'un genre et non d'un système de jugements lisible dans chaque texte.

Bien sûr, le Prix s'offre à l'adhésion, il cherche à concilier fait et valeur, il présente comme un donné valeurs acquises et impératifs naturels, il s'appuie sur un consensus préalable ${ }^{3}$, mais si l'on peut théoriser sur la nature idéologique du Prix, comment envisager le passage vers les textes?

II serait plus rentable de retenir une des fonctions essentielles du Prix, qui consiste à fournir aux lecteurs une matière prédigérée. Le Prix nomme ce qu'il faut distinguer d'une production générale et discerne, dans le produit, ce qui est le plus apte à retenir l'attention du lecteur. Le produit parvient au consommateur dans un emballage qui oriente la lecture, règle une fois pour toutes ce qu'il faut entendre de ce discours mis en marché. La fonction idéologique d'emballage assurerait au texte son déjà-lu. Elle est déjà reconnaissance, garantie de l'euphorie du décodage ultérieur que pratique le lecteur. "Toute une génération s'y retrouve", lit-on à propos 
du Temps des jeux de Diane Giguère ${ }^{4}$. Ce type de surcodage est le propre de l'investissement culturel qui contraint la lecture à venir.

Le texte primé est donc à la fois reconnu et masqué, et c'est pourquoi il est curieux de constater que du corpus retenu par nous, aucun titre n'est requis dans la chronologie de Maintenant', ni dans la colonne "Évéments culturels", ni dans "Livres, revues". Serait-ce qu'il y a en fin de compte, sinon une idéologie, du moins une vision des choses propre au Prix du Cercle? Un ensemble de critères plus ou moins ordonné, cela va de soi, mais quels rapports entretiendrait-il avec l'idéologie, et avec laquelle? Laissons pour l'instant la question pendante. Imaginons, par exemple, que si le Prix ne trahit pas d'emblée une idéologie dans ses choix, hors d'une certaine conception du monde fondée sur la rentabilité, la valeur, le profit, les textes pourraient en revanche marquer leur origine, leurs conditions de production.

«Tout groupe social, nous dit Jacques Grand'Maison ${ }^{6}$, tente de donner cohérence à sa situation dans toute son ampleur. II se bâtit, à cette fin, une vision de lui-même, de son milieu et du monde, un système d'explication, de normes, de valeurs qui dans un contexte historique donné, influence les attitudes et les comportements, les moyens et les fins que les membres de ce groupe partagent. »

Cette définition, qui recoupe celle de G. Rocher, plus haut, permettrait de considérer le texte comme construction idéologique (tenant lieu d'une idéologie), car le roman lutte pour sa cohérence et se fabrique un milieu et un monde. S'il se veut explication de normes et de valeurs, qu'en est-il de son influence sur les attitudes et les comportements? Le roman prend fin à la lecture, là où se termine le circuit marchand, et il serait aléatoire de risquer ne fût-ce qu'une indication sur son rôle effectif dans la société.

Poursuivons plutôt avec J. Grand'Maison : “L'idéologie, dit-il, implique une lecture particulière du passé, une interprétation du présent et une projection dans l'avenir ". Ces indications sont a priori assez conformes au schéma romanesque, mais, ajoute-t-il, "elle perd sa fonction essentielle quand elle n'a plus son caractère provisoire, quand elle devient un pur système intellectuel...", quand elle n'est plus que représentation. Nous dirons donc que le texte fige l'idéologie dont il pourrait se réclamer, qu'il en donne un signe appauvri, ou mieux : que l'idéologie se fige dans le texte et fige celui-ci. C'est ce que nous appellerons plus loin l'idéologique.

Le texte ne manifesterait donc que partiellement le système qui le produit. C'est ce que semble confirmer l'examen: le code qui préside à l'instauration du texte n'est lisible dans le texte que sous forme de fragments, d'extraits. Ce qui rend une lecture idéologique du texte, dans le sens d'un repérage d'une éventuelle idéologie du texte, éminemment contingente. C'est pourquoi on ne trouvera pas ici la recherche d'une affiliation du texte à des systèmes politiques qui ont marqué et marquent la société québécoise et dont on trouve un relevé chez $\mathrm{A}$. Bernard : nationalisme, con- 
servatisme, agriculturisme, libéralisme, etc. ou chez Grand'Maison : idéologie unitaire, néo-nationalisme, néo-fédéralisme, néo-capitalisme, etc. ${ }^{7}$. Ceci ne veut pas dire que toute référence à une instance productrice spécifique soit exclue, mais il est difficile de préjuger des discours qui régissent le discours romanesque de chacun des textes. Nous disons "des discours", comme A. Bernard, qui, dans le cas particulier de la société québécoise, tend à favoriser la thèse de plusieurs idéologies partielles concurrençant l'idéologie dominante, "s'il y a une idéologie dominante", précise-t-il, confirmant notre mise en garde quant à l'assignation automatique des textes à un système extérieur donné. Si ses trois éléments de définition de l'idéologie seront exploités plus loin (description, explication, proposition d'orientation), Bernard n'en affirme pas moins que «les idéologies se révèlent au niveau des collectivités (...) dans les écrits et les discours des porte-parole et leaders de ces collectivités ${ }^{8}$. Une telle assignation pourrait gratifier le texte d'un statut étranger à l'analyse textuelle ou littéraire, mais à la fois et peut-être contradictoirement tout texte manifeste un espace culturel, une culturalité, qu'il revient à l'analyse idéologique de dégager.

\section{De l'idéologie dans le texte}

Il faut aussi distinguer l'idéologie explicite de l'implicite. Nous parlons ici d'un repérage de surface, l'explicite étant l'apparition de contenus manifestement produits par (ou annexables à) une idéologie isolable hors du texte. Dans le corpus délimité, le seul cas de ce surgissement est celui du Poisson pêché (Georges Cartier).

Par idéologique, nous entendons le travail de l'idéologie dans le texte, c'est-à-dire une élaboration qui tend à normaliser un discours qui est un déjà-lu se réclamant d'un dire neuf. L'idéologique ne serait donc pas à lire comme manifestation d'un "système de représentations", mais visible comme indexation d'un discours à un registre extra-textuel. II s'agit ici du discours romanesque, qui échappe à toute tentative d'étiquetage ou de réduction au strict discours politique. Celle-ci négligerait ce qui fait justement l'intérêt du romanesque : lieu possible de tous les discours. II ne s'agit pas de nier l'origine du romanesque ou sa fin: le texte est traversé par l'idéologie, qu'il s'agisse de la texture proprement dite ou de l'appareil du livre, produit à décoder dans l'euphorique reconnaissance du code ${ }^{9}$.

Nous avons dit indexation; c'est donc voir le texte "comme un réseau de références "10, plutôt que comme discours se réclamant explicitement d'un extra-texte social. Il est pourtant délicat de trancher. En effet, que fait donc le discours sinon, comme l'idéologie, restituer "cette continuité et (...) produire l'unité sans cesse menacée du discours et de la réalité " ${ }^{11}$ ?

Le discours romanesque répugnerait à se démarquer clairement dans une société, sauf exception. Sur les cinq romans envisagés, aucun ne peut être d'emblée rangé là plutôt qu'ici, être classé “ réactionnaire" ou "révo- 
lutionnaire". Le seul qui semble traduire une option de ce genre ne le fait encore que dans les règles du romanesque. Disons pour l'instant du Poisson pêché qu'il pratique une indexation "virulente" par opposition aux autres. Voilà pour ce que nous choisissons d'appeler «l'idéologique explicite".

Pourtant, comme roman, Le poisson pêché "manifeste" également l'implicite, c'est-à-dire qu'en tant que discours il se constitue aussi par le jeu de l'idéologie dans le texte. Ce n'est qu'à la lecture que le jeu au sens plein apparaît: dans l'euphorie, à la fois saturation et ouverture.

\section{Appréhender le texte}

Le pluralisme idéologique que semble privilégier la société québécoise pourra donc surgir çà et là, mais notre projet n'est pas de le traquer. Notre corpus retient cinq romans, et que fait le roman sinon parler de luimême? all faut bien, rappelle $C$. Duchet ${ }^{12}$, que le roman commence en se signalant comme tel."

Nous avons déjà parlé d'indexation, nous parlerons également de «séries d'indices qui, plutôt que de signifier, désignent et indexent »13. Le texte est inséparable d'un référentiel, mais cet effet de réel est effet de texte, idéologique. Le texte renvoie au texte, mais alors que dire de l'indexation? C'est là toute l'ambiguïté de l'idéologique qui se désigne pour mieux se voiler.

Le hors-texte dont parle Duchet n'est donc pas une réalité extérieure, mais un produit du texte mis en place par l'incipit, et sa perception, si elle suppose un passé/passif culturel ${ }^{14}$, implique une codification particulière, un partage : un accès de part et d'autre à un répertoire.

D'autre part, le titre, la première et la dernière phrases, $s^{\prime}$ ils sont autant de repères, comme la jaquette et la couverture, ne distinguent pas le texte du hors-texte, mais de l'extra-texte, c'est-à-dire de l'ensemble des discours diffus que tient la société. Et si ces marques "ont déjà parlé le texte, déjà situé son contenu et son mode de lecture ${ }^{15}$, on peut y voir autant d'éléments générateurs du hors-texte, comme on l'observe avec Amadou.

Les marques d'indexation, fonctionnant à la manière de signaux, sont, pour les plus courantes, le titre, la préface, le préambule, l'incipit. Ph. Hamon y ajoute les citations, les exergues et les «embrayeurs d'isotopie» de Greimas, les personnages particuliers, les noms propres, les clichés et descriptions... ${ }^{16}$.

Dubois voit dans leur énoncé la preuve que le texte est réductible, soit à un projet (en amont), soit à une interprétation (en aval), ce qui revient à dire qu'ils constituent l'essentiel du texte. "Se forment alors, dit-il, des îlots de condensation" qui titularisent le texte ${ }^{17}$. C'est surtout à la description qu'il songe, insérant ses îlots dans la séquence narrative pour lui 
donner son poids de réel. II en va de même du cliché, qui raffermit l'indexation, l'ancrage à l'hors/extra-texte. L'unité menacée dont parle Ansart se consolide dans ce cadrage du discours qui guide et contraint la lecture, mais la facilité également.

Au système des idées reçues répondent les motifs stéréotypés, et nous avons le répertoire, notion qui appelle celle d'extrait. L'idéologique du texte se donne en effet à lire comme "citation" d'un répertoire institutionnel 18 . Mais si l'on retient que la citation indexe le discours, on ne peut y voir d'obstacle à la lecture.

Avec elle, "le discours paraît se confondre avec le bon sens", elle authentifie et explique le divers, l'anomalie en même temps qu'elle articule le texte et lui assure sa cohérence ${ }^{19}$.

II est donc normal que, manifestation du code, la citation soit itérative et restreigne la liberté du texte, qu'elle le fasse tendre à l'univocité, mais cette codification signalétique n'est asservissement qu'en vue de la reconnaissance. La forme idéologique du texte n'est rien d'autre que ce déjà-lu auquel se raccroche le texte, sa conformité au lisible, comme le signale D. Racelle-Latin, qui récuse justement au contenu idéologique (que nous appelons l'explicite) l'importance que d'autres ont pu lui donner ${ }^{20}$.

Il faut encore noter que l'idéologique (l'idéologie dans le texte) privilégie nécessairement la forme assertive, apodictique (évidence de droit, non pas seulement de fait), ce qui lui permet d'échapper à l'épreuve de la falsification, ni réfutable ni dépassable ${ }^{21}$. Dans le même ordre d'idées, le discours, par ses traits idéologiques, s'imposera comme exploitation des ressources langagières et se constituera autour de mots prestigieux, relais de l'extra-texte, mais aussi modalisation de la lecture, et notons par avance la connotation «poétique» d'un certain vocabulaire qui traverse Amadou.

\section{Le temps des jeux (Diane Giguère)}

Le roman se signale comme tel. Le temps des jeux porte en effet la souscription "roman», renforcée par le nom de la collection, "Les jeunes romanciers canadiens". Nous sommes ici au stade de l'emballage : ce qui permet de servir le texte au lecteur avec l'effet désiré.

Les éditions Laffont livrent au public français un produit éprouvé dans un marché-cible, garanti déjà par l'attribution d'un prix, doté d'une antériorité intertextuelle: Bonjour tristesse, et authentifié : «toute une génération s'y retrouve ». Ce discours d'emballage cite la presse qui lit dans le texte un déjà-vu. Le public français verra ses idées sur le Canada-français, sa jeunesse et sa littérature entièrement bouleversées. L'emballage informe: 


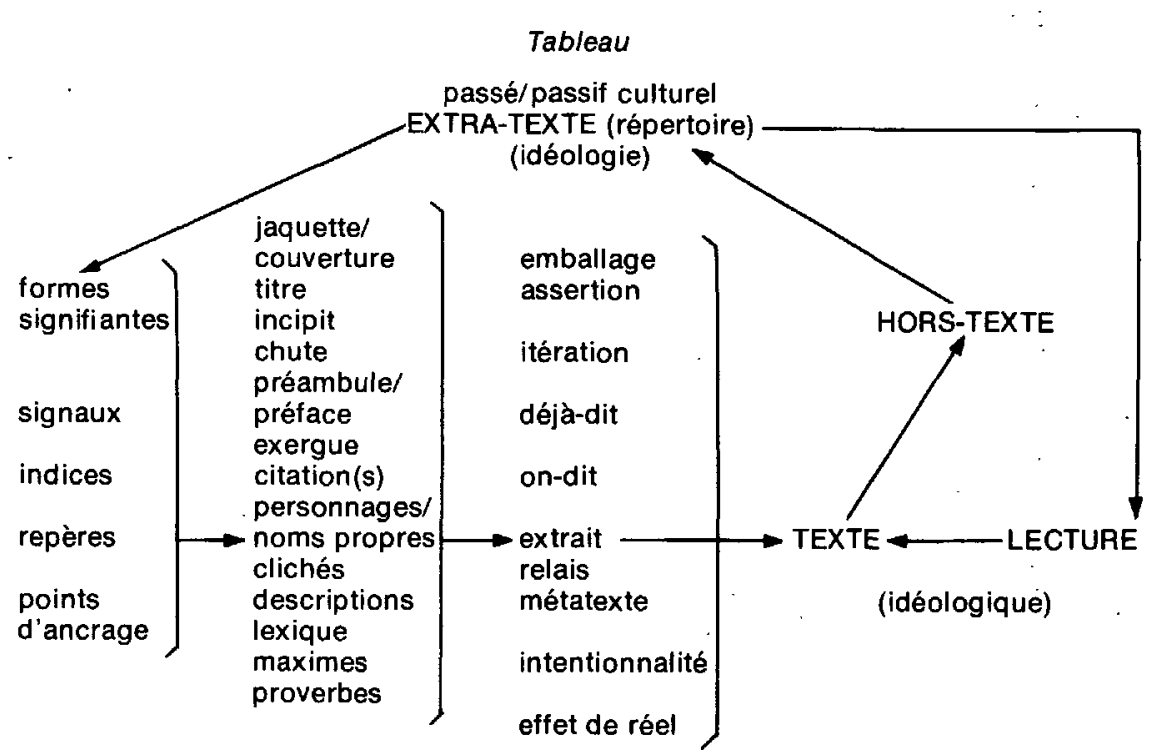

Le tableau ci-dessus récapitule les divers éléments examinés et renvoie partiellement à celui de Ph. Hamon ${ }^{22}$.

"quelque chose se passe au pays de Maria Chapdelaine" - la Révolution, mais modalisée: cette "révolution" (les guillemets sont dans l'original). L'œuvre est à lire comme un signe de ce quelque chose: elle est d'emblée indexée à l'extra-texte, avant même que s' inaugure le discours romanesque.

Cette fonction d'indexation appartient en propre à l'incipit auquel il revient de poser l'antériorité du texte, comme l'emballage pose l'antériorité d'une lecture (par le jury du prix, par l'éditeur). Mais c'est dans l'incipit qu'est le plus sensible l'arbitraire du texte, d'où la nécessité d'une part de l'indexation: le discours se raccroche à d'autres discours ou les invoque, et de l'autre, d'un protocole de lecture ${ }^{23}$.

L'incipit nous ouvre "la fenêtre naturaliste " ${ }^{24}$ et un horizon d'attente. $A$ ce bloc narratif doit succéder un bloc descriptif. La fenêtre s'ouvre sur la ville, mais également sur le mode éthique qu'annonce le titre. La fenêtre ouverte est un "débouché »: la mise en place de l'acte que dessine le corps qui oscille. Le suicide ainsi annoncé (horizon d'attente) s'énoncera en meurtre.

L'incipit est irréfragable. Mis en place du discours romanesque, il ne se discute pas; assertif, il est le type même de l'énoncé idéologique, plénitude incontestable.

L'apparition du descriptif aporte au narratif sa caution; la description encadre l'action, 
«...autour des grandes bâtisses qui longeaient le port. Prise de vertige

(...) à la croisée. Les rues désolées s'étendaient..."

L'objurgation morale s'offre en «citation» parée du déictique : “C'était aujourd'hui qu'il fallait mourir...» L'explicitation réitère le diffus conjugué de la morne description et de l'inquiétante narration. En rupture, la «citation " marque une indexation d'intentionnalité, et se donne à lire comme élément du protocole. Le temps des jeux étant fini, le drame commence, avec son dénouement : la mort. "Que c'était loin le temps des jeux..." (p. 9). Ainsi se réitère l'énoncé premier, référant le discours à son projet - le romanesque.

La "citation", on le voit, se donne pour modèle la maxime, ie recours aux valeurs. Le discours romanesque se pare du discours moral où jouent les culturèmes obligés. “On ne peut pas être si laid et ne pas en souffrir», (p. 42).

Dans les dialogues, la maxime se déploie à son aise, et se substitue au romanesque. L'ici livre le discours comme prélevé à un extra-texte et se donne comme extrait de l'on-dit. Ce n'est donc pas seulement la description qui a un effet de réel.

Le cliché est évidemment le type même de l'extrait d'un discours général, p. 82 : «... le charme et la tristesse de toutes les petites villes», p. 185 : «...la tête en feu, puis d'un pas décidé...», p. $35:$ «...comme un adolescent qui a honte de son corps" - et assure un maximum de lisibilité, une sémantisation accélérée ${ }^{25}$.

Cependant la maxime atteint sa pleine efficacité quand elle circonscrit et authentifie un personnage, comme p. 153: «Dans le fond, les professeurs n'ont aucun avenir, du moins pas-toi. » - où l'indexation au général "dans le fond" cohabite avec le modalisant marquant l'intégration au récit, «du moins".

Notre option de lecture, fragmentaire, peut sembler éluder la problématique du texte posée par l'emballage, mais cet emballage énonce l'explicitè du texte : la révolte contre l'autorité, la tradition, la quête du père, éternel absent du roman québécois (lieu commun, mais "vrai " comme.la plupart des lieux communs de la critique), c'est en quoi notre démarche, soucieuse de démarquer les zones de surgissement de l'extra-texte, soulève d'intéressantes questions. En effet, si le texte a le vertige de son autonomie, si sa fiction le gêne au point qu'il se raccroche sans cesse au déjàdit, n'est-ce pas précisément parce qu'il ne peut que mimer cette révolte, en livrer une allégorie, toute contrainte (ou contrite)?

Un des exemples les plus probants de la lecture d'un métatexte ${ }^{26}$ pesant sur le texte se trouve p. 171: “D'abord je vais quitter la maison..." où se pressent les clichés «valise prête», "seule solution", "sans grande conviction", "en se tordant les mains". 
II n'y a pas en réalité de moment privilégié pour l'extrait: il se donne libre cours dans le roman. L'à quoi bon de la chute, (pp. 207-8-9.) clôt à point le texte au seuil de son asphyxie par un hors-texte qui au terme de sa course n'a plus de raison d'être.

L'ultime itération du protocole livré par le titre : «C'est au temps des jeux qu'il aurait fallu être aimée... », n'y change rien. La gratuité du romanesque, dans son effort d'être déjà-là, n'en est que plus sensible.

\section{Amadou (Louise Maheux-Forcier)}

Amadou est riche en emballage : deux rabats, un texte en p. 4 de couverture. Notons du premier rabat: le piano, la musique, la littérature, les poèmes. Ces fragments du discours enrobant sont autant d'indications limitatives, contraignantes du hors-texte où se déploiera le discours romanesque. Le deuxième rabat synthétise ce discours dans une formulation mémorisable: “Ce thème», c'est le lesbianisme, innommé et transcendé en un "roman poétique, tendre et sensuel». Ce protocole facilite l'accès au texte par une citation exploitable.

L'exergue, citation de Tagore par laquelle le discours se réclame d'un garant, inaugure la lecture par une indexation poétique et musicale : le protocole en est consolidé. Le romanesque lutte sans cesse pour sa cohérence, avons-nous noté, et c'est ainsi que la mise en place du hors-texte est à saisir.

L'incipit pose une constatation irréfutable pour le lecteur, dont le déploiement du texte ne sera qu'une longue explicitation authentificatrice. Ce fait accusateur, «Je l'ai tué », marque l'arbitraire du texte et se donne à lire, à la césure entre le hors-texte et le récit, comme prétexte au discours qui s'amorce.

L'utilisation des points de suspension, effet de rupture, se signale comme effet de réel, mais aussi de l'incohérence d'un projet original dans un genre réglé comme le romanesque, d'où la nécessité de caractériser le sommeil de la victime par un connotateur plein et réaliste, d'où encore l'obligation de poser l'indiscutable: «ll y a une drôle d'odeur dans la maison..." Autour du je de la narration s'agglutinent les petits faits quotidiens, surgis de l'extra-texte.

Marquée dans son origine indéfinie, l'anecdote en rupture, sous forme de "citation», renforce la véracité de la situation du discours et en signale l'incohérence. "ll y avait... " Le souvenir du grand-père "fait vrai" dans le même ordre d'indexation. Au présent de l'odeur correspond le passé (biographique) du narrateur. L'antériorité posée par l'acte relaté (le meurtre) est entérinée par le relais de l'instance de la mémoire. Le récit reçoit sa justification - il vient après-coup pour expliquer, cautionner et malgré les heurts typographiques et les sursauts du discours, il se déroule inélucta- 
ble, selon le protocole qu'il se constitue. Aux points de suspension vont correspondre les décrochements (blancs) qui condensent le passé en îlots de sens qui devront, pour figurer leur cohérence menacée et marquer l'unité romanesque, s'indexer fortement sur l'extra-texte.

Ces îlots s'amorcent presque tous (le texte produit ici une de ces règles) par une indexation, parfois double comme dans le cas de l'épilogue ( $p .155)$ : «Alors, il y a ... L'alors marque le lien (la continuité) avec les fragments précédents et réitère le projet de conclure annoncé par le terme épilogue, tandis que l'il y a s'inscrit dans le système général du texte (cf. pp. 15-16, 22-23). Ce système se construit en contre-partie du hors-texte programmé comme le déroulement mnémonique et appartient au vertige de l'indexation qui traverse le texte inquiet de sa "relative autonomie", comme l'idéologie qui se réclame du fait, dans l'obsession de la valeur qui ne peut s'assurer qu'en posant l'évidence par le déictique (cf. pp. 12, 24, 25, 35, 39).

Les marques d'indexation introduisent l'élément textuel prélevé sur le texte culturel : “Alors, j'ai rencontré la Musique... », suivi de l'obligatoire explicitation, hyperbolique: "Tous les violons du monde se sont mis à chanter." L'indexation est surtout spatiale: le hors-texte qui se construit à la lecture possède ses références privilégiées, repères et indices d'un discours cultivé : cathédrales de France, clochers de la Forêt Noire, monastères de Yougoslavie (p. 17), Ophélie (p. 21), Rouault (p. 23), Alexis Zorba (p. 25), Matisse, Van Gogh, Cézanne (p. 31), Gide, Green, Montherlant (p. 117), etc. Espace culturel, touristique, littéraire, pictural: l'appel aux codes est constant et leurs marques prolifèrent pour garantir le discours menacé dans son projet.

Tous ces signaux, rapportables à leur discours d'origine, font peser sur le texte un métatexte et assurent la transitivité.

Le figement du sens s'opère par l'entremise des clichés, éléments de la lisibilité, commettre un grand péché (p. 39), faire ses gammes (p. 46), qui signalent une réitération du protocole (l'interdit sexuel, la musique), mais aussi la mainmise de l'on-dit sur le texte : «...tout n'est pas si simple." (p. 61), "Chaque chose à sa place..." (p.67), et l'intervention autoritaire du sens commun : "on ne reçoit jamais (...) c'est toujours autre chose..." (p. 68) ou de l'évidence, “Voilà!» (2 fois p. 74). Ces points d'ancrage ont une fonction de relais dans le texte: «Voilà! le décor est planté et voilà le cœur de mon histoire..." (p. 77) qui signale la «Deuxième partie ».

La citation est une mise en place et une caution : «mon mal du pays, c'est le visage d'Anne...».(p. 63), “C'est l'image de mon pere que je cherche” (p. 65). Le répertoire rompt le texte pour mieux l'asseoir: il l'installe dans la réalité illusoire de l'extra-texte.

L'énoncé romanesque se construit non seulement par indexation ou prélèvement au répertoire, mais par exploitation des formes culturelles nécessairement lisibles: "la fourmi prévoyante" (p. 93), dont l'assertion, 
forme du savoir pratique, du bon sens: "C'est une question de vitalité" (p. 95) et la modalisation «je crois» ne peut que confirmer l'effet d'adhésion, de la fonction phatique inséparable de la transitivité.

La dénomination (assertive) emprunte la forme de la maxime : "L'amitié est une chose merveilleuse...» $(p .96)$ ou offre le résumé du propos: “...c'est une œuvre d'art!" (p. 98).

La référence à l'art passe par les culturèmes obligés, le déjà-dit soit du texte, soit de l'extra-texte : “c'est l'œuvre d'art (...) superbement inutile» (p. 105) ou encore de la mythologie: «Nous avons cassé la croûte dans un bistrot..." (p. 103) ou du savoir : “...connais-tu ce conte de Maupassant...» (p. 126), Botticelli (p. 149).

L'incohérence est un effet de texte résolu par le surgissement des extraits du répertoire, l'itération de schémas descriptifs ou narratifs, comme le couple arbre-Julien, qui tous deux engraissent, ou le couple Anne-Sylvia, qui garantit la sensualité annoncée et qui réitère le thème du feu signalé par le titre Amadou, repris dans l'ultime effort de cohérence à la chute du texte qui annonce l'embrasement (p. 151). La cohérence clôt le discours, tandis que l'odeur de l'incipit est sémantisée au maximum dans sa polysémie: odeur des roses pourries et de la vie saine et normale, non sans une dernière indexation au discours littéraire: «Pirandello... Chacun sa vérité... » (p. 157).

Jalonné par la culture et l'acceptable, que reste-t-il d'un discours qui posait l'originalité et l'incohérence en conditions de sa production? Traversé par le lexique prestigieux de l'art, écrasé par les extraits du répertoire de l'on-dit, tient-il sa gageure? Sa nécessité est-elle autre que celle de ses garants?

\section{Histoire galantes (Bertrand Vac)}

Par le relevé des indexations ou des clichés, nous nous bornons à mettre en évidence les servitudes du genre romanesque. La démarche peut sembler limitative et paraître négliger ce qui fait l'originalité d'un texte donné. À repérer dans un discours l'asservissement à un modèle, le risque est grand de manquer ce qui fait aux yeux de certains l'intérêt d'un récit. II est bon, dans ce cas, de rappeler que nous visons à cerner et à observer les articulations du texte sur l'extra-texte, et l'on a pu voir que si généralement l'indexation se faisait sur un extra-texte global (réservoir des discours possibles), la mise en place d'un hors-texte relevait du particulier. Le horstexte, garant restreint, est ainsi spécifique à un texte donné. II s'agit d'un aménagement limité et oligé dans le cadre de l'extra-texte conçu comme discours parallèle de la culture, condition de production et de lisibilité du texte. Cette "culture" est en rapport de relative autonomie aux autres instances de production et se définit mieux comme idéologie(s) par ses carac- 
tères de "tenant lieu». L'idéologie ne peut que simuler d'autres discours et les formes signifiantes qui lui sont empruntées ne peuvent signifier qu'elle.

Dans un texte qui ne se signale pas comme roman, qu'advient-il ?

L'avant-texte d'Histoire galantes nous rassure: "Toute ressemblance avec des personnes existantes ou ayant existé serait purement fortuite et tout à fait involontaire"s. L'indice de fiction livré par le titre est réitéré dans l'avertissement: on ne peut s'y tromper, mais l'indexation au "réel" est posée avec une égale clarté, avec en corollaire quelque chose comme: "la réalité ne dépasse-t-elle pas la fiction?" L'avant-texte pose un horstexte qui ne serait autre que la vie, dont le texte se veut reproduction. Nous sommes en plein romanesque réaliste, avec comme protocole la galanterie.

L'incipit consolide cette mise en place du quotidien probable, à la portée de toutes les imaginations : "Un beau dimanche, elle se rendit à la Montagne." Le hors-texte se dessine dans un temps (loisir) et dans un espace (l'univers montréalais, son lieu de promenade riche en aventures). L'arbitraire du texte est minimisé par une forte indexation au banal. La vraisemblance remporte une adhésion optimale, renforcée par le bloc descriptif qui réitère le "beau dimanche" dans sa polysémie (l'occasion, le béau temps) : douceur de la saison, le printemps - appel au culturème «saison des amours". L'effet de réel compense le filtrage (distance) opéré par le passé simple et l'imparfait.

L'authenticité est garantie par la prévisibilité, alors que seuls les dialogues jouent de la méprise (une fois codée l'incommunicabilité ville/campagrie, autre culturème, lieu commun). Le lexique est ici celui des rencontres fortuites, caution globale d'un effet de réel maximal, en même temps que d'une lisibilité sans obstacle. Si l'horizon d'attente se solde par une disjonction au niveau narratif (les personnages se quittent comme ils se sont rencontrés, "comme ça»), la chute proprement dite, avec son attente à peine déçue, renvoie le lecteur à la réalité par un clin d'œil ( «ll la suivait »), signe que beau dimanche ou pas, la vie continue.

L'arbitraire restreint est le fait des sept autres récits, tous conformes au protocole galant : le sérieux est ici juste assez codé pour assurer un niveau lisible. Ces histoires de bonnes et de mauvaises fortunes amoureuses, malgré la forte indexation au réel, refusent le schéma coercitif du drame si fréquent dans le romanesque. Les schémas mis en place par Amadou et Le temps des jeux exigeaient un déploiement impressionnant de citations d'un métatexte romanesque qui, en relais, authentifiait le dire. Ici, l'indexation, si elle sous-tend une certaine perception culturelle, se fait sur le banal à peine aménagé, accessible à qui le veut bien, et si l'on peut admettre une continuité de construction, c'est celle de la légèreté, d'où parfaite adéquation du discours à son protocole.

Dans la diversité des Histoires, mêrne dans la deuxième, par lettres, on relèvera les mêmes modes d'indexation, les mêmes mises en place d'un hors-texte véridique et authentifiant, la même mise en œuvre de lexiques 
conformes, le même processus d'explicitation, les effets de réel assurés par la citation, le cliché, la description. La facticité, par exemple, du personnage de Margot (II) est programmée en bavardage, en inconscience. La guerre n'est là que prétexte à aventures, même si (indexation/extrait) "Les termes guerriers (lui) viennent tout naturellement. "L'assimilation du personnage aux grandes amoureuses de l'histoire (pp. 42-43) procède de la citation et du lexique prélevés dans le répertoire de l'amour comme valeur universelle. Et quand le texte se déplace géographiquement, il est indexé en conséquence (p. 45), Mexico, Acapulco, Pepe, Conchita, Puerto Vallarta. Sémantisation accélérée et explicitation : «T'ai-je dit qu'il me fait la cour? Aïe! »

Avec la troisième "histoire galante", la métatexte pèse du poids du lieu commun. II s'agit là de la première aventure d'un niais, que déniaise sa logeuse. Provincial arrivant à Montréal, il est pataud à souhait, comme l'explicite le texte à propos du dépucelage: "Au fond, il ne le désirait pas vraiment." "La vie d'étudiant" est codée tout au long du texte, dans sa double acception, comme en témoigne le "ll a passé brillamment tous les tests» (p. 83). Cette exploitation du lexique se démarque dans l'extension sémantique, au niveau narratif, de la logeuse voulant être traitée par le «docteur» (p. 59), avec explicitation du type proverbial : «Après tout, c'est son métier. " La transparence onomastique est à noter dans le cas de Jolicoeur, nom à prendre au pied de la lettre, qui est opérateur de lisibilité. À lui revient le rôle de narrataire et de relais du savoir commun en matière de dépucelage. La fente (p. 67) dans le mur de la chambre est indexation spécifique au récit réaliste, la fenêtre étant le motif obligé du spectateur privilégié.

La quatrième histoire, avec son incipit : «Quand John Green ne s'amusait pas, il amusait", s'inscrit dans la même veine, par les mêmes marques. Citation, cliché, explicitation, transparence (Green-vert-verdeur), descriptions encadrantes, etc. Le lexique est celui des histoires salaces et de la drôlerie. On joue dans le connu, le banal même, lu comme plaisanterie (airs de vierges outragées, p. 89, la mésaventure - «cet affreux incident ", etc.)

Transparence onomastique encore dans la cinquième «mésaventure», Guillaume le coureur se nomme Courseul et la vierge imprenable, Malencort, le «depuis des siècles" (p. 93), habillé de guillemets, indexation à un type de rapports sociaux, est suivi de son explicitation, comme "cet affreux incident ", traduit par le narrateur en mésaventure.

Dans le discours réaliste, le personnage commande une triple description, en général par emprunts aux divers savoirs: sphère d'activité, lieu d'activité, activité proprement dite ${ }^{27}$; le lieu est décrit (p. 98), l'activité (p. 110 et 113). L'achat de la Buick sollicite le savoir mécanique, mais if faut noter ( $p .102-103$ ) que la galanterie subvertit la mécanique, comme la pose, prétexte à déployer le projet : concurrence de savoirs - l'approche érotique et son lexique métaphorique disputent la préséance à la voiture comme à la peinture. Les lexiques figent les personnages dans des zones de sens, garantissant la plausibilité des comportements : la vierge (prin- 
cipes religieux, salut de l'âme, p. 107, en état de péché mortel, p. 119), le coureur («En amour, tant qu'on n'est pas arrivé à ses fins, toutes les ruses sont bonnes », p. 109).

La sixième histoire s'amorce sur une constatation à caractère proverbial, tirée du répertoire de l'on-dit qui relaie les hors-textes déjà exploités et marque une itération propre: le texte se redit et cite le répertoire. Le déjà-dit textuel est signalé en particulier par le "Colony" déjà donné en repère au $\|{ }^{e}$. Les parcours sont tracés, seules les connexions varient. Nul beson de poser une antériorité construite, ici l'effet de réel ne se fonde pas sur le petit fait vrai, ou la description scrupuleuse. On puise allègrement dans le répertoire du connu et du quotidien.

Au VIle l'adolescence appartient au passé partagé, comme les laitiers. L'apprentissage de la virilité, s'il ne se fait pas toujours dans une camionnette, n'exige aucune référence à un extra-texte déterminé, sinon le nécessaire surgissement de l'idiome vraisemblable (têteux, p. 153, run, p. 157, fait une blonde, p. 161, chum, p. 171, etc.). L'inscription de l'on-dit n'en est que plus forte: quoi de plus accessible, de plus vrai? «Avec les femmes, on paie toujours" (p. 163).

Avec la VIIle et dernière "histoire galante", on observe la même économie d'indexation, mais également la même construction par prélèvement au répertoire et la même itération du posé antérieur. Marcel le séducteur, Marie la tigresse : «Inutile de répéter ce qu'ils se dirent d'abord, c'est connu» ( $p .186$ ). Le cliché participe à l'économie du texte; inutile de chercher un discours original quand le stéréotype est rapide, efficace : «...tous les espoirs étaient permis. Alors, fidèle à une tactique ancestrale (...) déboires de la vie conjugale" (p. 188).

La chute signale bien la théâtralité de l'ensemble des Histoires galantes. Sortie théâtrale, partie remise ( $p .151)$, rideau retombé ( $p .119)$ : la galanterie est un jeu, et le texte est codifié en ce sens. L'on-dit constitue ainsi le métatexte essentiel de ces contes, mais loin d'écraser le texte, il lui imprime un mouvement qui facilite la lecture. Nul besoin d'un hors-texte élaboré si la «vie» est prise à témoin. De tous les discours culturels, les "vérités éternelles " sont les plus sûrs. L'évidence, conciliation du fait et de la valeur, de l'être et du paraître, n'est-ce pas là en plein l'idéologique?

\section{La corde au cou (Claude Jasmin)}

La corde au cou nous parvient "bardée" d'emballages: biographie, bibliographie "minimum exacte" à l'intention des étudiants - le texte se signale en classique. Cette profusion paratextuelle fonctionne à la façon d'un filtrage, mais surtout dresse le texte en autorité, en le situant dans le temps.

L'antériorité, analogue à celle d'Amadou, marque l'acte répréhensible, mais aussi signale le romanesque, par le drame, et l'arbitraire du texte. Le 
lecteur n'a pas à soumettre le texte à l'épreuve de vérité, il se soumet: de lui, on exige l'adhésion. Le narrateur et ses marques sont autant de signaux phatiques. L'anaphorisation précède en quelque sorte le dire. Et le crime antérieur se répétera, en protocole, pour se fermer sur une euphorique justification culturelle: «ll est consolant de savoir que j'achève d'être mal élevé. " L'indexation à l'extra-texte privilégie une citation du répertoire anti-militariste (p. 10) qui assure la cohérence et pose un hors-texte vraisemblable.

Le roman s'inscrit dans une durée qui lui est extérieure: "le remords ", «continue», «morte», «bien avant» (p. 7). C'est à cette durée qu'il prélève sa matière, c'est cette durée qui constitue l'histoire parallèle (" tout a eu lieu », « tout s'est passé »...) qui sert de caution au discours, assure l'authenticité. D'où la nécessité de marquer l'antériorité, de la réitérer. Le remords, que chaque "méfait" ou crime vient expliciter, donne au texte sa cohérence, facteur de lisibilité. Sur le mode de l'assertion, "Ce fut la dernière fessée imméritée (p. 27), de l'invérifiable, "Nous connaissions..." (id.), de la connivence, “Quand je vous dis..." (id.), le hors-texte dessine son champ ouvert sur l'extra-texte (enfants précoces, école de réforme, etc.), crédible, dans la conformité du paraître à l'être. Bavard, le discours se désigne et se commente «...cet ami, purement fictif, de Montréal» (p. 59), dans une large itération, «le vieil homme de mes vacances d'enfant délinquant ( $p$. 83), se noue autour de la corde du protocole (remords = culpabilité $=$ châtiment) dans une transparence onomastique (Cordier, p. 117), et le cliché : “finement ciselée» (p. 118) “...s'était empreint de tristesse...» (p. 119), "la main au collet" (p. 120), comme l'effet de réel (eau tiède de la piscine, p. 7) authentifie le drame, c'est-à-dire le romanesque.

Ainsi se constitue un mode de lecture réaliste, conforme, où l'explicite peut surgir sans crainte ("...pays, tant occupé par ses poussées économiques (...) pas de place pour les idées (...) les sentiments (...) désordre... " p. 11). "Amérique nouvelle», pp. 105-106, mais intégré au discours, il se borne à commenter, expliciter le drame «...jeune homme sans éducation, il n'est pas de notre monde!" (p. 109). L'extrait à sémantisation accélérée ( échapper à la vie, oublier qui je suis», p. 112) inscrit de larges pans du discours : Tokyo/ "rue grouillante de monde», Cannes/ "chaloupes", Milan/ "pizza" (p. 112) chanteuses/ " hit parade», "opéra» (p. 132), manufactures de vêtements pour dames/ «rue Bleury» ( $p$. 143), télévision/ «images» ( $p$. 161). L'extrait sous forme de maxime («histoire d'amour qui finit mal») traverse le texte (p. 93-95, les plaies d'Égypte, les fables citées) relayant le protocole ( J'étais un meurtrier précoce dont la destinée était bien clairement tracée" ", p. 12).

Le tissu du texte, multipliant les marques d'indexation, tend à une lisibilité que trouble à peine la phrase courte, autre marque de la sémantisation accélérée: dès l'incipit le texte souligne sa finalité ( «avoir mal cité le mauvais auteur", p. 12) : la lecture qui l'englobe et constitue son simulacre de référent, le hors-texte, caution de sa normalité, de sa prévisibilité 
et, somme toute, de son efficacité. Pressé d'en finir, le texte court à son terme en sautant les obstacles (les astérisques, marques de rupture toujours compensées par l'itération et l'anaphorisation): “j'achève», en se répétant : "mal élevé» (p. 163) et ces décrochements s'offrent en esquive de l'itération maximale, comme si le texte cherchait à échapper à l'étranglement de son discours par le stéréotype.

\section{Le poisson pêché (Georges Cartier)}

Le poisson pêché est le seul des cinq romans, avons-nous dit, à manifester l'explicite idéologique, c'est-à-dire qu'il donne à lire une série plus ou moins organisée d'énoncés de type ouvertement emprunté à une zone spécifique de l'extra-texte: en l'occurrence, la politique. Ce qui ne revient pas à dire que Le poisson pêché soit politisé ou «exprime" une idéologie rattachable aux tentatives de nomenclature faites par $A$. Bernard et $\mathrm{J}$. Grand'Maison 28. Au contraire, Le poisson s'annonce en indexation à une région de l'extra-texte apparentée à celle d'Amadou, la poésie, si l'on s'en tient à une lecture directe de l'exergue (citation de Henri Michaux), mais une lecture de biais (un biais qui passe par le texte) nous livre, en plus de l'explicitation de l'origine du titre, une hypothèse sur ses conditions de production: l'exil. L'exil du poisson hors de l'eau tient lieu de l'exil du narrateur hors de son territoire, de son terroir, de son terreau ( « racines", p. 108). L'exil, l'habitat, le territoire: la repossession de son pays se pratique sur le mode synecdochique: à travers Joan, c'est son passé/passif culturel qu'il étreint. Pour le narrateur, le mode figuré va jusqu'à falsifier ce bilan : il invente à Joan une généalogie ( «malgré son nom anglais, elle descend bien de mes ancêtres", p. 10).

L'exergue ne dénonce-t-il pas également l'arbitraire de son origine? La figure traverse tout le texte, guidant la lecture et la contraignant à un décodage des métaphores. Fiction, le roman l'est doublement : romanesque par le répertoire mis en œuvre, il marque dans la métaphorisation la quête illusoire et contradictoire de l'unité canadienne. "Canadienne ", lit-on, pour Colombie-Britannique (p. 11). Il ne s'ag it pas là d'un simple élément de mise en place du hors-texte "géographique": on peut y voir la première articulation du mensonge aux prises avec les aspérités de son discours. Appareil complexe qui se déploie en trois temps: le texte se donne daté, dédicacé et contemporain du récit. Indexée sur une chronologie collective, instituant une destinataire, la fiction embraye sur le petit fait vrai («...je note ces mots... $)$, et pose un décor : la chambre, lieu de l'intimité, réitération de la communication privilégiée, la confidence.

Joan a dès l'abord un statut discursif: c'est par son relais que se constitue le hors-texte; elle apparaît en déclencheur d'extraits - on assiste dès ce signal à un déferlement de citations territoriales: l'Ouest, les Rocheuses, l'Alberta; et historiques : La Vérendrye, indexation du passif culturel ("...que je croyais oublié», p. 11). Ces prélèvements au répertoire ne se 
font pas sans contraction d'une dette: ce qui permet le repérage de la citation réside dans sa forme - cliché. Le répertoire déverse ainsi dans le texte un îlot de clichés : «surgir du néant ", "créaient, littéralement ", "vaste plaine», «sommets vertigineux», etc.

L'indexation sur une histoire parallèle (l'Histoire dès relations QuébecCanada - figurée par Joan et le narrateur) ne fait pas de doute, et non plus la solution qui s'imposait (historiquement): l'exil. "Entre la France et le Canada, puis-je encore trouver ma patrie?" On ne peut être plus explicite. Piège encore du discours emprunté : le Canada, choix équivoque - «Foutaise, les beaux projets d'enfant prodigue, les pensées nobles de nationaliste borné! " L'explicite se donne toujours en citation.

La territorialité se constitue en métatexte et remplit l'espace textuel : par analogie, elle donne lieu à un inventaire de la France touristique, pp. 158-161, “Suivez le guide! de préférence le Guide Bleu. " L'explicite submerge le discours, condamné aux stéréotypes pour parler du pays «aux proportions démesurées" (p. 164). Culturème encore: le parallèle Français-Québécois (p. 130) obéit au modèle observé chez Bertrand Vac, au détriment du Français. Cette valorisation se passe de commentaire.

Lieu d'énonciation, l'exil ne tarde pas à trahir son indexation véritable: "Pourquoi m'exiler au Canada?" (p. 75). Le métatexte territorial ne manque pas d'apparaître avec ses marques référentielles: solitude, p. 45, "l'eau nous entourait de toutes parts", p. 227, "grand fleuve», p. 23, «peuples jeunes", p. 23, etc.

On observe, d'une part, cette élaboration géopolitique du texte et, de l'autre, le surgissement de l'irréconciliable: “Qu'y a-t-il de commun entre l'Est et l'Ouest du Canada?" (p. 22). "En vain», lit-on dans la citation du poème qui clôt le texte. L'effort de reconquête, constitutif d'une assumation du passif culturel, se solde par un échec, indexé cette fois sur un poème québécois (canadien, dit le texte). A l'intertexte français se substitue le québécois: le bilan serait-il positif, le mensonge romanesque livrant sa vérité et Céline succédant à Joan?

\section{Le romanesque québécois des années soixante : en quête d'une idéologie?}

Le texte compose avec l'idéologie, écrit J. Dubois ${ }^{29}$. Nous avons tenté ici de montrer comment, par quels moyens privilégiés et à quelles fins, l'idéologie composait, inversement, le texte, en relevant ses marques, en mettant en évidence quelques-unes des articulations idéologiques du texte. Incipit, citation, protocole, anaphorisation, explicitation, métatexte, horstexte, autant de notions qui permettent un repérage du texte pour en proposer une interprétation qui loin de se substituer aux autres devrait les éclairer. 
Après avoir ainsi marqué l'idéologique des textes, leur inscription dans un système plus général qui les constitue et qui se porte garant de leur évidence et de leur nécessité, il serait bon sans doute d'avancer de quelles idéologies ils participent, ne fût-ce qu'à titre d'hypothèse.

La corde au cou et Le poisson pêché à quatre ans d'intervalle s'indexent en clair, explicitement, à une territorialité, caractère propre du nationalisme, selon A. Bernard ${ }^{30}$, dont la mutation en indépend antisme, la restriction au Québec, se situe dans les années soixante. Référent limité dans La corde au cou, le territoire devient, dans Le poisson pêché, la grande question, quel qu'en soit le détour, par le biais de l'exil, et l'on y discerne les conflits résolus par des solutions fictives, romanesques. Cet exil, paré d'autres motifs, constitue l'essentiel de romans comme Amadou ou Le temps des jeux : le passé/passif extratextuel contraint le texte à l'imaginaire, A l'inlocalisable - le discours se réclame d'un genre à défaut d'un lieu. Le nulle part du Temps des jeux répond à la France culturelle d'Amadou, que récuse et ridiculise Le poisson pêché. Histoires galantes combine sans vergogne l'ici et le là-bas, annule les conflits, comme si, en 1965, la question ne se posait plus.

"Là-bas, vous ne voyez rien. Nous, ici, on voit tout le pays! "Le père Ubald de La corde au cou (p. 155) confirme la subsistance de l'agriculturisme jusque dans les années 50-60 et Le poisson pêché manifeste les transformations qui déchirent le Québec en quête d'une identité. Si Le temps des jeux et Amadou prônent par leur indexation un manque comblé fictivement par le mythe d'un espace culturel continu (France $=$ Québec), La corde au cou et Le poisson pêché se réclament du néo-nationalisme fluide, polymorphe et plurivalent que définit J. Grand'Maison ${ }^{31}$, propre à la "Révolution tranquille", tandis que Histoires galantes ferait état, dans son apparente indifférence idéologique (explicite), d'un croisement réducteur entre le néo-fédéralisme et le néo-capital isme signalés par le même auteur: pluralisme, et cette absence de malaise serait une souscription docile au statu quo.

La chute normalisante de La corde, du Temps, d'Amadou, du Poisson et d'Histoires nous rappelle cependant qu'il s'agit là de romans, de solutions fictives, parties intégrantes d'un corps d'énoncés possibles, le genre romanesque, vestige bien portant de l'idéologie bourgeoise triomphante $\mathrm{du}$ $X \mid X$ siècle. D'où l'impossible indépendance que manifestent diversement les textes étudiés ici. Et n'est-ce pas cette appartenance contradictoire, cette existence par procuration (dans l'espace culturel français) que signalait et récompensait le Prix?

Ne doit-on pas voir dans cette ultime correspondance (pluralisme = romanesque) le repliement inévitable et confortable sur l'idéologie «éternelle», transhistorique (Althusser), alors que les idéologies spécifiques ne sont pas en mesure de mobiliser les écritures et les textes? En revanche, avec la déchéance du Romanesque, la “révolution tranquille» deviendrait-elle Révolution? 
Confortable, disons-nous : les émergences possibles d'une conscience nationale se résorbent dans un genre neutralisant, normalisateur, acceptable, où les conflits se résolvent en discours fictifs, comme autant de tranquillisants, administrés au public par l'instance régulatrice, le Prix, délégation du Pouvoir.

1. G. Rocher, Introduction à la sociologie générale, $2^{\mathrm{e}}$ édition, Montréal, Hurtubise-HMH, 1972, Tome 3, p. 375.

2. Ce sont, dans l'ordre de leur parution, de C. Jasmin, La corde au cou, Montréal : CLF, 1960; de D. Giguère, Le temps des jeux, Montréal : CLF, 1961 (nous utilisons toutefois l'édition Laffont de la même année); de L. Maheux-Forcier, Amadou, Montréal : CLF, 1963; de G. Cartier, Le poisson pêché, Montréal; CLF, 1964; et de B. Vac, Histoires Galantes, Montréal : CLF, 1965. Le Prix couronne un manuscrit retenu en finale par un jury composé de critiques littéraires et de personnalités en vue du monde littéraire.

3. F. Dumont, Les idéologies, Paris : PUF, 1974, pp. 113-114.

4. Au dos du volume.

5. Numéro double 137-138 (juin-septembre 1974, pp. 11-12), dans lequel la revue présente un tableau général des événements importants des dernières décennies.

6. Dans "L'Église et les idéologies au Québec", Relations, $N^{\circ} 343$, Novembre 1969, p. 293.

7. Voir Bernard, A., La politique au Canada et au Québec, Montréal : PUQ, 1976, pp. 100-120; art. cit., pp. 294-295.

8. La politique au Canada..., pp. 98, 123.

9. J. Leenhardt, “Modèles littéraires et idéologie dominante", Litterature, $N^{\circ} 12$, décembre 1973, Paris: Larousse, p. 19.

10. J. Dubois, "Code, texte, métatexte", Littérature, $\mathrm{N}^{\circ} 12, \mathrm{p} .3$.

11. P. Ansart, Les idéologies politiques, Paris: PUF, 1974, p. 23.

12. C. Duchet, "Pour une socio-critique ou variations sur un incipit", Littérature, $N^{\circ} 1$, février 1971, Paris : Larousse, p. 8.

13. Dubois, art. cit., p. 6.

14. Duchet, art. cit., p. 12.

15. Id., p. 6.

16. P. Hamon, "Note sur les notions de norme et de lisibilité en stylistique", Litterature, $\mathrm{N}^{\circ} 14$, mai 1974, Paris : Larousse, p. 122.

17. Art. cit., p. 10.

18. J. Dubois, art. cit., p. 9.

19. P. Ansart, op. cit., p. 19.

20. Danielle Racelle-Latin, "Lisibilité et idéologie, le cas du texte célinien", Litrature, $\mathrm{N}^{\circ} 12$, p. 87.

21. P. Ansart, op. cit., pp. 18, 24, 25.

22. P. Haman, op. cit., «Notes sur les notions... », p. 121.

23. Voir J. Dubois, "Surcodage et protocole de lecture dans le roman naturaliste", Poétique 16, Paris : Seuil, p. 491.

24. Voir P. Hamon, “Un discours contraint », Poétique 16, p. 430.

25. Id., 440.

26. Dubois, "Code, texte, métatexte", pp. 8-9.

27. Voir Hamon, "Un discours...", p. 432.

28. Voir Introduction ici même. 
29. Dans L'Assomoir de Zola (société, discours, idéologie) Paris : Larousse-Université, coll. "Thèmes et textes", 1973, p. 105. Son chapitre 4, "l'inscription idéologique" recoupe tes articles cités et met en œuvre l'essentiel des notions du présent article.

30. A. Bernard, op. cit., p. 121.

31. Art. cit., p. 294 ; cf. A. Bernard, op. cit., p. 124. 